Dansk Ultralyddiagnostisk Selskab

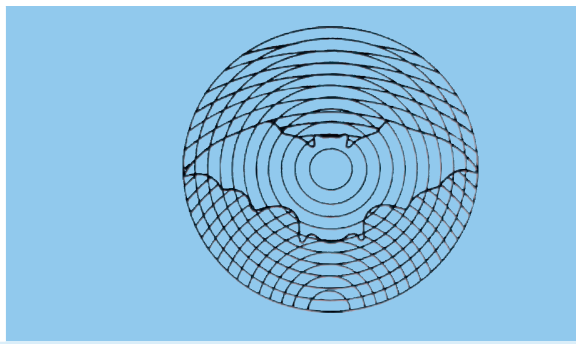

\title{
Cardiac vector flow imaging
}

\section{Kristoffer Lindskov Hansen ${ }^{1,2}$}

\section{${ }^{1}$ Department of Diagnostic Radiology, Copenhagen University Hospital, Copen- hagen, Denmark \\ 2 Department of Clinical Medicine, University of Copenhagen, Copenhagen, Denmark}

Imaging of the cardiovascular flow is an integrated part of the cardiac exam in patients with congenital and acquired cardiac conditions. The conventional imaging methods are ultrasound, computed tomography, angiography, and magnetic resonance imaging. While ultrasound is cheap, mobile, real-time, and safe, the ultrasound mode used for flow assessment, Doppler ultrasound, is limited by angle dependency [1]. This implies that only flow in the direction of the ultrasound beam is evaluated. Vector flow imaging is a new real-time ultrasound method for angle independent flow assessment introduced by Jensen et al. [2]. The first studies of cardiac vector flow imaging have been conducted using a commercial ultrasound platform with a linear array transducer ( $\bullet$ Fig. $\mathbf{1}$ ), and the studies revealed that cardiac vector flow imaging is possible and useful [3].

The initial studies concerned feasibility of the method when applied intraoperatively in patients with normal and diseased aortic valve $[4,5]$, while the following studies explored advantages, limitations and possible applications of the method when applied intraoperatively on patients scheduled for cardiac by-pass surgery and aortic valve implantation [6-10]. Examples of vector flow imaging have been reported of blood flow in the normal and the congenital diseased pediatric heart using transthoracic ultrasound [11].

Among the findings were shown that the aortic secondary flow consists of a systolic



- Fig. 1 Vector flow imaging of the ascending aorta in longitudinal (left image) and transverse scan plane (right image) acquired intraoperatively. The real-time vector velocities are displayed as colored pixels on the B-mode image. The colors given by a 2D color bar define flow direction and velocity magnitude, and the superimposed vector arrows facilitate the flow interpretation.

and diastolic component both related to the primary antegrade flow and aortic valve stenosis [6, 7], that atherosclerotic plaques have a predilection for vessel locations with altered flow [7], that flow complexity of the primary antegrade flow of the ascending aorta is related to aortic valve stenosis [6], and that congenital heart defects alter the flow direction in the ventricles [11].

It is indicated that cardiac vector flow imaging can provide visualization of the complex flow patterns within the heart and great vessels, that conventional as well as new flow measures can be obtained with vector flow imaging, and that new information of normal and altered flow can be gained with vector flow imaging in healthy persons, in patients with congenital heart defects, and in patients with acquired cardiovascular diseases. Cardiac vector flow imaging may become a powerful tool for cardiovascular flow assessment.

\section{References}

[1] Jensen JA. Estimation of Blood velocities using ultrasound: A signal processing approach. New York: Cambridge University Press; 1996

[2] Jensen JA. A new estimator for vector velocity estimation. IEEE Trans Ultrason Ferroelec Freq Contr 2001; 48: 886-894

[3] Hansen KL. Cardiac Vector Flow Imaging; Copenhagen University; 2019: 1-77

[4] Hansen KL, Moller-Sorensen H, Pedersen MM et al. First Report on Intraoperative Vector Flow Imaging of the Heart among Patients with Healthy and Diseased Aortic Valves. Ultrasonics 2015; 56: 243-250

[5] Hansen KL, Pedersen MM, Moller-Sorensen H et al. Intraoperative cardiac ultrasound examination using vector flow imaging. Ultrason Imaging 2013; 35: 318-332

[6] Hansen KL, Moller-Sorensen H, Kjaergaard ] et al. Aortic Valve Stenosis Increases Helical Flow and Flow Complexity: A Study of IntraOperative Cardiac Vector Flow Imaging. Ultrasound Med Biol 2017; 43: 1607-1617 
[7] Hansen KL, Moller-Sorensen H, Kjaergaard ] et al. Analysis of Systolic Backflow and Secondary Helical Blood Flow in the Ascending Aorta Using Vector Flow Imaging. Ultrasound Med Biol 2016; 42: 899-908

[8] Hansen KL, Moller-Sorensen H, Kjaergaard J et al. Intra-operative Vector Flow Imaging Using Ultrasound of the Ascending Aorta among 40 Patients with Normal, Stenotic and Replaced Aortic Valves. Ultrasound Med Biol 2016; 42: 2414-2422
[9] Hansen KL, Moller-Sorensen H, Kjaergaard ] et al. Vector Flow Imaging Compared with Conventional Doppler Ultrasound and Thermodilution for Estimation of Blood Flow in the Ascending Aorta. Ultrason Imaging 2017; 39 (1): 3-18

[10] Hansen KL, Møller-Sørensen H, Kjaergaard ] et al. Vector Flow Imaging of the Ascending Aorta in Patients with Tricuspid and Bicuspid Aortic Valve Stenosis Treated with Biological and Mechanical Implants. Ultrasound Med Biol 2020; 46: 64-72
[11] Hansen KL, Juul K, Moller-Sorensen $\mathrm{H}$ et al. Pediatric transthoracic cardiac vector flow imaging - a preliminary pictorial study. Ultrasound Int Open 2019; 5: E20-E26 